\title{
IMAGINARIOS EN PUGNA. LA POLITIZACIÓN DEL CAMPO DE LOS ESTUDIOS ANDINOS EN EL PERÍODO FUNDACIONAL DE LA ETNOHISTORIA ANDINA CHILENA
}

\author{
IMAGINARIES IN STRUGGLE. THE POLITIZATION OF THE FIELD OF ANDEAN \\ STUDIES ON THE CHILEAN ETHNOHISTORY FOUNDATIONAL PERIOD
}

\author{
Carlos María Chiappe*
}

\begin{abstract}
En este artículo analizamos tres textos científicos de inicios de la década de 1970 centrados en la cuestión indígena del Norte Grande de Chile. En tanto consideramos que los mismos constituyen un campo propicio para indagar las relaciones entre ciencia y política, mediante su estudio buscamos exponer las rupturas y las continuidades de los imaginarios sociales que estos expresan. Nuestro interés principal es pensar la naturaleza, alcances y limitaciones de un conocimiento particular: el que se construyó en las comunidades indígenas del norte de Chile por medio de la relación entre las políticas de desarrollo y la práctica científica.

Palabras claves: Estudios andinos, ciencia aplicada, imaginario social.
\end{abstract}

In this paper three scientific texts from the beginning of the 1970's are analyzed, focused on the Indian issues from the Norte Grande of Chile. As we consider that they build a propitious field to enquire about the relationship between science and politics, through their study, we aim to expose the ruptures and continuities of the social imaginaries that they express. Our principal interest is to think about the nature, scope and limitations of a particular knowledge: the one built upon North Chilean Indian communities through the relationship between the development politics and scientific practice.

Key words: Andean Studies, Applied Science, Social imaginary.

\section{Introducción}

En un trabajo precedente (Chiappe 2014) analizamos el proceso de modernización de las ciencias sociales latinoamericanas apuntando que el mismo se vinculó con la geopolítica bipolar de la Guerra Fría, siendo una manifestación particular dentro del adelanto de las ciencias sociales mundiales en el que estas se revalorizaron al postularse que sus conocimientos eran indispensables para entender los problemas que obstaculizaban el desarrollo social y para poder accionar sobre ellos. De ese modo, intentamos pensar el citado proceso como la confluencia de variados fenómenos (institucionalización, internacionalización, regionalización, autonomización y politización) que le otorgaron al campo académico-científico regional una configuración ligada a la inserción periférica de Latinoamérica en el sistema capitalista mundial y centrada en la cuestión del desarrollo (Ansaldi 1991).
En este marco propusimos que, al menos entre 1960 y 1970 , el rasgo sobresaliente de los estudios andinos chilenos fue su participación en tareas de diagnóstico acerca de los ecosistemas y las comunidades rurales indígenas, con el objeto de sopesar el potencial de adecuación de los mismos a las reformas en marcha, en el contexto de las variadas políticas desarrollistas impulsadas e implementadas por el Estado nacional, pero relacionadas además con las demandas de la política global.

En relación con este tema, en este artículo nos interesa profundizar en el análisis de cierta producción científica escrita durante el gobierno de Salvador Allende, en donde es factible visualizar el interés de la época por el desarrollo de las comunidades andinas en el marco de diferentes proyectos políticos. Esta producción puede caracterizarse como anteproyectos de investigación en donde los autores explicitan su postura en relación con la forma en que las investigaciones respecto de la problemática indígena en el norte del país debían realizarse. * Universidad de Buenos Aires, Facultad de Filosofía y Letras, Sección Etnohistoria. Buenos Aires, Argentina. Correo electrónico:
carloschiappe@ yahoo.com.ar 


\section{Breve descripción de las fuentes}

En primer lugar, se hace preciso defender el hecho de que analizaremos una producción muy puntual, sin tomar en cuenta otros trabajos de los mismos investigadores. Como esto podría ser objetable, decimos que nuestro planteo se justifica en tanto no queremos analizar recorridos intelectuales, sino poner de manifiesto diferentes posicionamientos ideológicos insertos en la orientación predominantemente aplicada de los estudios andinos de esa época.

La primera de nuestras fuentes la tomamos de la republicación conjunta en 1973 de los números primero y segundo de la revista Chungara, siendo su autor el arqueólogo Mario Rivera. Esta está conformada por dos textos, uno que constituye el editorial de la revista (A manera de introducción: Hacia una política de desarrollo integral del Departamento de Arica), y otro que es un artículo del mismo número (Bases para planificar la investigación antropológicaarqueológica en el Norte Grande chileno). La segunda fuente es autoría de Freddy Taberna, geógrafo social, cuadro político del Partido Socialista y funcionario del gobierno de Allende que fuera fusilado por las autoridades de facto en Pisagua el mismo año de 1973. El trabajo que analizamos se dio a conocer en el Primer Congreso del Hombre Andino, un evento que se realizó en las sedes nortinas de la Universidad de Chile y que cristalizó el estado de un movimiento académico que había conocido un gran crecimiento por esos años (Núñez 2013). En él, además de la presentación de los avances en el estudio de las sociedades centro-sur andinas, se opusieron diferentes perspectivas relacionadas con la coyuntura política del país (Calogero Santoro 2010). La cuestión del etnodesarrollo aparece en forma central en la evaluación actual de los objetivos fundamentales del evento por parte de uno de los integrantes de la comisión organizadora (Núñez 2013: 95). En razón del golpe de Estado que aconteció pocos meses después -y que conllevó la censura de las ciencias sociales y la persecución de los investigadores-existe poco material impreso del congreso, pero pueden hallarse algunas de las contribuciones, como es el caso de la fuente que usamos aquí (Simposio No 4: El rol de la sociedad andina y el tránsito al socialismo. Informe: Algunas Condiciones Básicas para el estudio del tránsito hacia el Socialismo de la Sociedad Andina). Es factible destacar que, pese a la orientación disciplinar de los autores, ambos tuvieron la misma formación de grado en la Facultad de Filosofía y Educación de la Universidad de Chile, en donde accedieron (en 1963 Rivera, en 1968 Taberna) al título de Profesor de Estado en las Asignaturas de Historia, Geografía y Educación Cívica (Chiappe 2014 b).

El trabajo de Rivera presenta los lineamientos del "Proyecto Integral de Antropología de la Universidad del Norte" orientado a las comunidades indígenas del interior del Departamento de Arica. Sus objetivos son definidos como: conocer el mundo andino, diagnosticar los "problemas culturales" del Departamento de Arica y formular propuestas para políticas de desarrollo basadas en los puntos de vista antropológicos (Rivera 1973: 15).

Aunque el autor no haga una descripción sistemática de lo que él entiende por mundo andino, del análisis de sus fuentes y a base de las influencias que luego discutimos, podemos inferir que este refiere al ecosistema altiplánico, en donde los integrantes de las comunidades indígenas son parte de una entre varias de las especies biológicas coexistentes, pero que se diferencia de las demás en tanto detenta el mecanismo adaptativo supraorgánico particular del ser humano: la cultura.

El supuesto desde el que se parte es que los "problemas culturales" tienen como causa el "choque" entre dos entidades culturales: el mundo andino y el grupo de la urbe. Las relaciones sociales producto de este impacto se traducían en una discriminación del mundo urbano hacia el mundo andino, lo que llevaba al aislamiento sociológico de las comunidades indígenas $\mathrm{y}$, como efecto a largo plazo, a su desintegración. En este marco, la investigación buscaría determinar los "problemas fundamentales" que impedían la correcta implementación de las políticas de desarrollo. El autor hace hincapié en la necesidad de llevar adelante proyectos de investigación interdisciplinarios de larga duración acerca de las poblaciones indígenas (pasadas y presentes), ya que solo en la medida en que se pudiesen comprender los fenómenos culturales que formaban la realidad que se quería cambiar podría lograrse el desarrollo de la gente en su medio.

El proyecto tenía entonces una especial relevancia para toda política "inserta en una realidad cultural que desconocemos" y este se instrumentaría "con el fin de ir dando las bases de los futuros planes de desarrollo que fomenten la revitalización e integración del mundo andino" (Rivera 1973: 14-15).

Pasemos ahora al trabajo de Taberna. Su tema es el rol de las sociedades andinas en el tránsito 
hacia el socialismo. Sus objetivos son delinear la situación del campesinado andino en el norte de Chile y determinar la posibilidad de su integración en la vía chilena al socialismo del gobierno de la Unidad Popular.

Taberna argumenta que las investigaciones debían realizarse a partir de las propias concepciones e intereses de los pueblos andinos - cosa no negada por Rivera- pero mediante el método materialista dialéctico, única forma en que se podría dar cuenta de la conformación de las fuerzas productivas que constituían la base de la crisis regional.

De este modo se proponía estudiar el desarrollo del proceso histórico regional; la "matriz cultural andina" (entendida como la relación entre estructura y superestructura de tal sociedad, de la que se desprendía el mantenimiento de su "modo tradicional de vida"); las condiciones objetivas generales (explotación) y particulares (sus diferentes características culturales) del campesinado andino; las relaciones entre las instituciones burguesas y los pueblos andinos, medio por el que el capitalismo penetraba y desintegraba a la sociedad andina; $y$, finalmente, la forma en la que podrían darse las condiciones subjetivas para que se produjese una revolución cultural del campesinado cuando este comprendiese que la supervivencia de su matriz cultural y su propio desarrollo socioeconómico dependían de su incorporación al proceso revolucionario (Taberna 1996 [1973]: 48-53).

La relevancia de la investigación se centraba por tanto en su papel político, ya que el científico, al estudiar las condiciones objetivas de los pueblos andinos, ayudaría a generar las condiciones subjetivas necesarias para que los mismos pudieran lograr sus reivindicaciones dentro del proyecto nacional.

\section{Hegemonía en disputa: estructural-funcionalismo versus marxismo}

Indagaremos ahora los marcos de cognoscibilidad adoptados por los autores suponiendo que estos nos permiten no solo analizar cuestiones teóricometodológicas, sino también políticas. Esto es así porque los mismos están asociados a relaciones de hegemonía del campo científico situadas en contextos sociohistóricos particulares y porque, además, estos no se aplican en el vacío, sino sobre problemáticas concretas. En nuestro caso, la implementación de la Reforma Agraria y la Ley Indígena de 1972. Los diferentes marcos teórico-metodológicos, entonces, pueden dar razón de los posicionamientos políticos de los investigadores que los adoptan.

El análisis de las fuentes de Rivera nos lleva a proponer que su problematización, objetivos y metodología se encuentran comprendidos en los fundamentos de la ecología cultural y del procesualismo. El enfoque ecológico cultural (sensu Steward 1955) se expresa en la búsqueda por determinar la interrelación entre la tecnología y el entorno que es explotado mediante ella; en el interés por analizar los modelos de comportamiento de un área particular que están presentes en su forma de explotación productiva, la que depende tanto de la historia cultural como de la ecología del área; y en la intención de investigar en qué forma estos modelos de comportamiento modifican a otros aspectos de la cultura. Su metodología se sustenta en un abordaje holístico que permita interrelacionar los diferentes componentes de la cultura mediante el trabajo interdisciplinario entre la antropología social, la arqueología, la historia y la antropología biológica. Por lo demás, la influencia del procesualismo se hace notoria por la insistencia en que la arqueología es una ciencia antropológica cuyo fin es entender los procesos de cambio cultural; por la planificación de la investigación a partir de una hipótesis de trabajo; por la implementación de una metodología científica rigurosa y por la primacía de la interpretación de los datos por sobre la clasificación de los mismos. Esto concuerda con el contexto temporal de la fuente, momento en el que se estaba produciendo una profesionalización y renovación de la disciplina.

En tanto Rivera postula que los problemas culturales tenían como origen principal el "choque" entre el mundo andino y el grupo de la urbe, una crítica que es posible hacer es que la gran abarcabilidad de estos conceptos lleva a presentar a las sociedades como totalidades homogéneas en donde el individuo es un mero reproductor de la cultura. Además, subsisten en las fuentes la distancia cultural entre el investigador y los sujetos estudiados, entendida como una separación entre sujeto y objeto solo en donde es posible producir el conocimiento científico. Estas características, propias de la antropología estructuralfuncionalista, denotan cierto atraso, ya que para la época que tratamos se estaba empezando a generar el cambio del sujeto de estudio desde una alteridad radical a otra próxima, en el marco de los procesos de descolonización y del consecuente aumento de las "antropologías nativas" (Palestini et al. 2010). 
Otro elemento que aporta en el mismo sentido y que va a contrarreloj de los tiempos políticos del país es la opción por el uso de términos asépticos en el abordaje de las problemáticas sociales. Cuando se tratan las consecuencias de la relación entre el mundo andino y el grupo de la urbe se opta por hablar de los efectos de la "economía moderna" sin entrar en explicitaciones acerca de las relaciones de dependencia político-económica del sistema mundial. Así, el contacto con la "sociedad moderna" habría beneficiado en cierta forma a los grupos indígenas (educación básica, salud, bienes materiales), pero habría introducido al mismo tiempo y a veces por los mismos cauces factores de desintegración al desaparecer elementos culturales tradicionales, bien adaptados a la estructura económico-social de las comunidades (Rivera 1973: 13). Entendemos que el enfoque materialista no marxista de la ecología cultural permitía postular que los fenómenos que obstaculizaban el desarrollo eran producidos prioritariamente por un desbalance entre el medio ambiente y la acción cultural, lo que habilitaba excusarse de tomar una postura ideológica respecto de las causas de tales efectos.

Sin embargo, Rivera adopta un posicionamiento más explícito y ataca las políticas gubernamentales al señalar que las soluciones teóricas a los problemas reales no funcionarían si no se tenía un acercamiento empírico al "problema cultural" de base. El autor aludía así a la, en su parecer, equivocada forma de implementación de la Reforma Agraria, en tanto no se habían atendido las particularidades de las sociedades afectadas. El modo de remediar esto principiaba por entender que cualquier reforma de este tipo consistía en un cambio cultural. Alteraciones de tal envergadura precisaban, primordialmente, formar a la población. De allí el lugar importante que el autor asignaba a la educación en el proceso, aunque esta dilatase los tiempos de implementación, en contra de las modalidades de acción de los organismos de ejecución. Pero, como para poder educar primero había que aprender de las comunidades, esto llevaba a la necesidad de implementar proyectos survey, ya que solo en la medida en que se pudiesen comprender los fenómenos culturales que formaban la realidad sobre la que se quería operar podría fomentarse el desarrollo económicosocial. En este sentido, al criticar el hecho de que, aunque se desconocían las concepciones nativas en materia de derecho, se aplicaban medidas legislativas de alcance nacional que negaban el derecho a la autodeterminación de los pueblos indígenas, Rivera embestía también contra la implementación de la Ley Indígena de 1972.

Por otra parte, existe una relación sólida entre el par opuesto mundo andino-grupo de la urbe con el de costa-sierra de Valcárcel (1927) y de Mariátegui (2007 [1928]). La persistencia de la tesis de la dualidad estructural como forma interpretativa del funcionamiento de las sociedades latinoamericanas sería criticada por Rodolfo Stavenhagen, ya que -en opinión del sociólogo mexicano-en estas sociedades los sectores "atrasados" constituían en realidad el polo de aprovisionamiento en donde los sectores "dinámicos" de las mismas absorbían los recursos que usaban en beneficio del sistema capitalista global. No existían entonces sociedades duales sino colonialismo interno (Stavenhagen 1975: 41).

En tanto los problemas culturales impedían la implementación de una adecuada política de desarrollo, esto nos lleva a pensar que, en última instancia, la responsabilidad de la falta de integración al desarrollo nacional recaía en las características culturales de los indígenas. Para comprender qué entendía el autor por problemas culturales creemos que es útil tomar el concepto de traducción intercultural, forma en que en la Antropología clásica (sensu Malinowski 2000 [1922]) era entendida la labor disciplinaria. Debido a que el problema de fondo era cómo podía ayudar la Antropología a integrar al proceso de desarrollo a los pueblos originarios en un panorama que destacaba por la ausencia de datos confiables, la categoría de problemas culturales representaba el desafío de traducir al idioma de la ciencia las particularidades de los pueblos originarios que podían operar como un impedimento a los planes de desarrollo para que, de esta forma, se pudiesen implementar políticas con sustento real.

$\mathrm{Si}$, en Rivera, el interés por analizar las relaciones entre agrupaciones sociales a base de las problemáticas existentes impide pensar a las comunidades indígenas como inmunes al cambio, esto queda en parte matizado por la consideración de que aquellas se aislaban sociológicamente en respuesta al desprecio étnico. Se minimiza así la larga historia de interacción entre las comunidades y los grupos dominantes y, además, se opaca el hecho de que estas estaban integradas a la sociedad nacional en el marco del sistema capitalista mundial.

Además, aunque discrepando con la propia opinión del autor al ser preguntado al respecto 
(Mario Rivera, comunicación personal 2014), creemos que su idea de tradición lo acerca a un tópico del pensamiento de Alejandro Lipschutz (1937). Recordemos que la cultura para este último era un todo constituido por elementos materiales y simbólicos interdependientes, una tradición orgánica vivida que permitía desarrollarse al grupo humano en su ambiente. En tanto Rivera tiene por objetivo "determinar los momentos diagnósticos en ese desarrollo cultural que habían asegurado su identidad mediante la tradición cultural" (Rivera 1973: 15); y que, además, busca entender el proceso de cambio respecto de la hipótesis de que este y la tradición son mecanismos complementarios (Rivera 1973b: 47), podemos argüir que, de forma similar a Lipschutz, la cultura se actualiza constantemente en un proceso en el que se va constituyendo una tradición que es típica de cada agrupación social. Esta concepción dinámica lo coloca a cierta distancia de la forma en que la cultura era pensada por la Antropología clásica, como un ente caracterizable por un conjunto finito de rasgos. Pero la similitud se corta aquí si aceptamos que Lipschutz dio razón del cambio social por medio del concepto de transculturación, consistente en procesos vividos por todos los pueblos al entrar en contacto con otros grupos y que suponen el traspaso de elementos culturales entre los mismos en el contexto de las economías que cada grupo desarrolla. La transculturación es la experiencia más asidua de la historia humana y, por tanto, no puede ser pensada en forma unívoca, ya que esta puede tanto afectar negativamente a las comunidades indígenas como también propender a su revitalización. En el trabajo de Rivera, en cambio, los beneficios del contacto con la "sociedad moderna" son minimizados en relación con los fenómenos de desintegración que han experimentado las comunidades al desaparecer los elementos culturales tradicionales adaptados a la estructura económica social (Rivera 1973: 13). Este posicionamiento se enmarca en una visión de la sociedad consistente en un conjunto de subsistemas tan íntimamente solidarios que una variación en algunos de ellos lleva a un "desbalance ecológico" que implica el fenómeno de desintegración étnica.

Por todo lo anterior, creemos que es válido juzgar que las fuentes tratadas presentan los elementos propios de la Antropología estructural-funcionalista: su orientación comparativista, universalista y científica (Stocking 2002) que son también fundamentos de la Ecología cultural y del procesualismo.
En clara oposición nuestro otro autor, Freddy Taberna, rechaza toda investigación que estudie las particularidades de las sociedades andinas sin tomar en cuenta el proceso de desarrollo histórico regional en el marco del sistema mundial capitalista. Esta perspectiva, al negar implícitamente la lucha de clases, apoya las medidas desarrollistas de corte reformista opuestas a un cambio social revolucionario.

Taberna toma como propio el planteamiento central de los Siete Ensayos de Mariátegui (2007[1928]) consistente en que el problema indígena es de tipo económico-social. A partir del mismo podemos entender la insistencia en evitar la categoría "indígena" y el uso de otras homólogas entre sí (sociedad andina, campesinado andino, hombre andino, sociedad campesina tradicional, masa campesina, campesinos proletarizados, campesinado indígena, pueblos andinos y comunidades campesinas) que sirven tanto para situar a estos grupos sociales en un determinado medio (el andino) como para caracterizarlos según su actividad económica (campesinado). Esto va en sintonía con el enfoque clasista y es también un rechazo al relativismo estructural-funcionalista, lo que adquiere una claridad meridiana cuando se trata el tema de la discriminación. Si Rivera (1973) alude al desprecio étnico sin entrar en detalles acerca de las causas de este fenómeno, Taberna, en cambio, entiende que este se da para favorecer la explotación social. Su negación a utilizar la categoría de "indio" es afín a las ideas del momento de Guillermo Bonfil Batalla (1972) en el marco de un planteamiento de la problemática indígena en donde era central situar a las masas indígenas como parte del proletariado.

Si Taberna enfatiza primero la necesidad de analizar el estado actual del campesinado en el marco de las relaciones capitalistas, plantea luego también la utilidad de analizar el desarrollo histórico regional. La conquista de América es entendida como un choque económico-cultural que impactó a las instituciones indígenas, a la vez que como precondición de la futura penetración del sistema capitalista, ya que esta implementó un régimen social basado en las relaciones entre dominadores y dominados, relaciones que subsistían aún y a las que se alude bajo las categorías de explotadores y explotados, haciendo hincapié en la base económica de tal dominación.

Más allá de su común matriz cultural, Taberna también señala que se debía indagar las particularidades de las comunidades indígenas, pero siempre 
partiendo del reconocimiento de las condiciones objetivas generales de explotación, con la finalidad de ayudar a que se concreten las condiciones subjetivas necesarias para la incorporación del campesinado a la revolución socialista, en sus propios términos y desde sus propias concepciones. Se trataba de integrar las particulares concepciones del desarrollo indígena que no se opusieran a los fundamentos socialistas (tarea al parecer no improbable en tanto la propia matriz cultural era de carácter comunitaria) $\mathrm{y}$, al mismo tiempo, propender a una modificación gradual de las instituciones que, por la "deformación" producida por la penetración capitalista, no estuvieran en consonancia con estos fundamentos. En esencia, el cambio buscado era el que iba desde una economía precapitalista contaminada por el capitalismo hacia una economía socialista. Y este cambio podía entenderse como una potenciación y mejora de la matriz cultural "socialista" de los pueblos originarios.

El capitalismo aparece en Taberna (como en Rivera lo es el "mundo urbano") como el principal agente del cambio, en un marco social formado por comunidades con leves diferenciaciones internas, atadas a una "tradición" poco propensa a las modificaciones en virtud de su base económica agropecuaria de carácter cíclico y conformadas por sujetos que tenían una percepción "subjetiva e irreal" del actual proceso chileno (Taberna 1996 [1973]: 60). Es ejemplificador el párrafo en el que se tratan los efectos perniciosos de las nuevas necesidades impuestas por el capitalismo por medio de las alteraciones producidas en el tráfico caravanero, desde los viejos patrones transhumánticos que permitían el aprovechamiento colectivo de una vasta red de recursos, hasta la actual utilización del mismo para el tráfico de estupefacientes. Aquí, el autor erra en no reconocer, de modo análogo a lo hecho por Rivera, la capacidad adaptativa de los pueblos originarios a los múltiples escenarios históricos y el papel que juega el acaparamiento de los bienes materiales en las relaciones intra e interétnicas. Está claro que ya para la época la Antropología marxista se estaba realizando preguntas nuevas que no implicaban abandonar el planteamiento clasista.

Aunque cuestiones como las anteriores nos inducen a pensar que la característica eminente de la fuente, su objeto político, lleva a aplicar un tanto esquemáticamente los conceptos marxistas, en otro momento el autor resalta un interesante aspecto que lo desliga de la aplicación mecánica. Cuando
Taberna dice "En general la teoría marxista no ha sido bien utilizada para analizar el rol superestructural de la sociedad andina" (Taberna 1996 [1973]: 55) y propone, por tanto, investigar la particular relación entre estructura y superestructura que se evidencia en el maridaje entre las festividades y el ciclo agropecuario; no significa esto que la base material dejase de determinar en última instancia a los componentes superestructurales, sino que estos últimos dominaban las relaciones entre la economía y el mundo simbólico, cosa que establecía una taxativa diferencia con la sociedad capitalista. Existía entonces una "íntima relación entre la supervivencia del trabajo comunitario como organización de infraestructura, y la fuerte manifestación religiosa superestructural" (Taberna 1996 [1973]: 55). Creemos que este punto de análisis encuentra su complemento en las ideas de Maurice Godelier, cuando este plantea que en las sociedades precapitalistas "las relaciones de parentesco funcionan como relaciones de producción, relaciones políticas, esquema ideológico. El parentesco es pues a la vez infraestructura y superestructura" (Godelier 1978 [1968]: 178, el resaltado del autor). En efecto, los ayllus, unidad de parentesco básico de las comunidades andinas, son el marco en el que las unidades domésticas intercambian trabajo y cooperan en el manejo de los recursos y, por tanto, el medio en donde la estructura económica se activa mediante principios de reciprocidad vehiculizados por prácticas rituales (Stern 1982). En este sentido, desde la perspectiva de Godelier, sería correcto el planteo de Taberna en torno a complicar la relación entre base y superestructura. Sin embargo, el dominio de la estructura económica no estaría operado desde la religión misma, sino desde las relaciones sociales por las que cualquier ideología se organiza y, además, estas mismas relaciones se hallan imbricadas en los dos órdenes del modo de producción.

Remarquemos finalmente que la fuente también está influenciada por algunas de las ideas de Alejandro Lispchutz, introductor del marxismo en el medio científico chileno. Cuando se trata la necesaria emergencia de las condiciones subjetivas que harán que los pueblos originarios, al tomar conciencia de su rol histórico, se sumen a las transformaciones en curso, se lo hace en términos de una revolución cultural que deberá producirse en los mismos y a la que la investigación marxiana está llamada a hacer un gran aporte. Pensamos que este término es deudor del de resurrección indoamericana, forma 
con la que Lispchutz retrató la reivindicación económica y cultural de las masas indígenas. Ambos conceptos aluden al término de la desintegración de las comunidades andinas y al inicio de su revitalización (sensu Rivera 1973) en el marco del proceso revolucionario que Lipschutz (1937) entendió como una futura y necesaria reorientación político-social del continente americano.

En conclusión, creemos que el examen de los marcos teórico-metodológicos de ambos autores da razón de sus posicionamientos políticos. Esto queda bien ejemplificado en la diferente postura que estos adoptan alrededor de la Ley Indígena de 1972 y de la Reforma Agraria, que estaba en aplicación desde 1962. Rivera las entiende como imposiciones estatales que no toman en cuenta los valores propios del mundo andino y que, por lo tanto, están destinadas a fracasar. En contraposición, Taberna las exalta abiertamente, al incluir los problemas campesinos en el panorama político de un país desestabilizado por el fascismo. En ataque abierto a enfoques "cientificistas" que hacían énfasis en los métodos de recolección y análisis, declinando explicitar su posicionamiento ideológico en el contexto político del momento, Taberna tilda de reaccionario a cualquier tratamiento de la problemática de las comunidades campesinas que no partiese de un análisis marxista, ya que entendía que este servía de sustento ideológico a las políticas de desarrollo afines a la burguesía y opuestas a la vía chilena al socialismo.

Es claro que este análisis no debe llevarse a extremos. Rivera comenta que

...con Freddy Taberna no comulgábamos políticamente hablando, pero a la hora de plantear políticas de desarrollo coincidíamos en muchos aspectos. Por esta razón, siendo Freddy director de Planificación y Desarrollo de la Primera Región del Norte en la época de Allende, siempre me invitó a reuniones de trabajo y seminarios precisamente porque, a pesar de ser independiente políticamente hablando, teníamos bastantes coincidencias y lográbamos un entendimiento respecto de cómo conducir el desarrollo en la región (Mario Rivera comunicación personal 2014).

Por tanto, desde la visión actual de Rivera relativo a las relaciones político-personales entre él y Taberna -se entiende que sin posibilidad de contraste por el asesinato del último- las oposiciones que tratamos y que se nos aparecen como irreductibles tienden a matizarse.

\section{Ciencia, política e imaginario social: aperturas y limitaciones}

En este apartado realizamos un análisis de tipo conclusivo acerca del modo en que el entramado entre ciencia y política posibilitó -y al mismo tiempo limitó- la construcción del conocimiento científico en las fuentes analizadas. Posteriormente, y basado en lo anterior, pensaremos el papel de la naciente Etnohistoria andina en la generación de un nuevo tipo de saber de los sujetos indígenas.

En principio diremos que la ciencia, tanto como cualquier otra producción social, incorpora una visión particular de cómo es y cómo debería ser la sociedad y una disputa con ideales opuestos. Aunque, visto así, el trabajo científico tiene siempre un cariz político, en opinión contraria, autores como Mario Bunge (2000) o Gregorio Klimovsky (1997) han argumentado que debe distinguirse a la ciencia como expresión del conocimiento humano del uso social que se le dé a este último. Estas ideas se fundan en una valoración del conocimiento científico como "neutral" y "potencialmente beneficioso". Queda en manos de las instituciones encargadas de aplicar los progresos el que esto se haga en favor del género humano. En apoyo de nuestra postura, tomaremos a Eduardo Marí (1990; 1991), quien sostiene que la distinción entre una "ciencia neutral" y el mal o buen uso que la sociedad haga de esta es falsa, en tanto oculta las condiciones sociales, políticas y económicas que influyen en la producción científica; el hecho de que el científico no es un ente individual, ya que la ciencia es una práctica social $\mathrm{y}$, finalmente, que no hay neutralidad en la observación científica. Para Marí no se puede reducir lo científico a la mera teoría porque toda teoría incluye en su aparato conceptual sus condiciones de aplicación y, por tanto, la misma está hecha en función de resultados esperables. En consonancia, Oscar Varsavsky (1969) opina que no existe práctica científica apolítica y autónoma porque la ciencia está inmersa en un campo de poderes que inhiben su desarrollo "libre". Por tanto, este autor nos propone realizar una ciencia politizada guiada por el ideal de una autonomía relativa. Una ciencia que, a pesar de las limitaciones tanto estructurales 
como del propio conocimiento, pueda reservarse a decidir acerca de sus finalidades.

Ahora bien, si lo político es constitutivo de la práctica científica. Si cada uno de nosotros, implícita o explícitamente, incorpora en su quehacer un determinado posicionamiento, ¿cómo debemos abordar los ideales contrapuestos que expresan nuestras fuentes? Nuestro punto de partida aquí es el señalado por Weber (2002 [1922]) en relación con que la ciencia jamás podrá resolver una contienda entre sistemas de valores que son como "dioses antagónicos". No obstante, lo que sí puede ayudarnos a hacer es a preguntarnos en qué modo una determinada forma de abordar un problema de estudio desde un particular "sistema de valores" o ideal político abre ciertos horizontes y limita otros. $\mathrm{Si}$, como podemos argumentar tomando a Gadamer (1988), la investigación es el lugar en donde se encuentran diferentes mismidades y en ella la pregunta es el elemento original que crea el espacio de comunicación que posibilita la construcción del conocimiento mediante el diálogo entre puntos de vistas particulares: ¿en qué modo una matriz liberal que piense a la sociedad como sumatoria de individuos cada uno de los cuales procurando su bien individual logran el bien común ayuda a captar, pero también oblitera, las problemáticas que forman parte de la realidad de las comunidades andinas? (v.g. agencia, etnicidad, relaciones de dominación). Lo mismo vale para el abordaje marxista, vista la sociedad como un campo de lucha de clases. Ya que el lugar desde donde preguntamos preanuncia la respuesta que obtendremos, la admisión del sesgo que esto conlleva nos permite plantear la mayor limitación de los enfoques analizados. Aquella que es fruto de su autonaturalización, de la negación de su historicidad inherente en tanto productos sociales (Castoriadis 1993). Sin embargo, que el sesgo sea constitutivo no imposibilita su corrección. Esto ya lo planteó Franz Boas (1987 [1929]) con la idea de kulturbrille, "anteojeras culturales" que todos llevamos puestas y que nos obligan como investigadores a la exigencia metodológica de revisar los propios condicionamientos.

Nuestras fuentes exponen la confrontación entre diferentes ideales en un momento de la sociedad chilena propicio para que la producción científica se desnudase de su aparente neutralidad ideológica. Estas pueden ser leídas como manifestaciones particulares de imaginarios sociales en una forma antitéticos y en otra semejantes. Refiriéndose a la misma realidad social, Mario Rivera nos propuso pensar el sentido que debía tener el hacer ciencia en una nación en desarrollo, Freddy Taberna en un país inmerso en un proceso revolucionario. Ambos coincidían en la necesidad de promover políticas fundadas en un conocimiento científico que incluyese los puntos de vista indígenas, pero se oponían en la forma en que el mismo debía construirse. Es detacable decir que ambas posturas son comprensibles, ya que, sin considerar su mayor o menor validez científica y tratando de suspender en esta evaluación nuestras simpatías políticas, estas respondían a los imaginarios en pugna de la época, los que aspiraban a ser de validez universal, ignorando su origen contingente.

Rescatamos de ambos autores la continuidad con la vocación indigenista de restituir derechos negados y abordar el rol de las masas indígenas en los agitados procesos nacionales. Siguiendo a Gunderman y González (2009), podemos concluir que lo negativo es que -pese a las intenciones manifiestas-minimizaron la agencia de las comunidades, toda vez que su actualidad fue pensada desde el impacto desintegrador que el choque con el capitalismo o con el mundo de la urbe había producido. Así, Rivera propuso estudiar los "fundamentos de la vida comunitaria que orientaban la conducta social y cultural" y determinar "las influencias del medio ambiente y de las fuentes de aprovisionamiento que incidían en los fenómenos sociales propios de cada comunidad". Su interés estaba puesto en entender cómo las fuerzas internas de las instituciones locales de la comunidad campesina indígena daban razón de la cohesión social. Por su parte, Taberna no indagaba a nivel de las pequeñas comunidades -lo que no quita que haya tenido contacto directo con ellas y se haya preocupado por sus intereses-, sino del espacio social mayor del que emanaba la "matriz cultural" del "hombre andino". Esta "vieja matriz", que era lo que definía la especificidad del sujeto indígena, daba cuenta de las continuidades materiales y simbólicas entre los pueblos extintos y presentes, ya que en razón de la pertenencia tradicional de la comunidad campesina al espacio andino esta llevaba en sí una matriz comunitaria o socialista "en germen" que la hacía compatible con el marxismo. En el primer caso, no se indagaba el marco sociopolítico mayor del que estas comunidades formaban parte; en el segundo, se homogeneizaba su variedad. El apolítico relativismo estructuralfuncionalista tendía a producir un conocimiento descontextualizado. La focalización en la denuncia 
de la explotación capitalista subsumía las dimensiones étnicas de las comunidades indígenas que no concordaban con la vía chilena al socialismo.

\section{Reflexiones Finales}

Aun sin considerar el trabajo de investigadores considerados pioneros -Cúneo-Vidal (Galdames 2009), José Toribio Medina (1882), Alejandro Lipschutz (Berdichewski 2004)- y a pesar de estar enmarcada en un territorio geográficamente periférico respecto del de sus desarrollos originales (Perú), la Etnohistoria andina chilena tuvo tempranos antecedentes. Esto debe relacionarse con la revolución historiográfica promovida desde ciertos espacios de la Universidad de Chile, visible como el paso de una historiografía centrada en los individuos y acontecimientos políticos a otra que enfocase los procesos y las estructuras sociales. Este hecho estuvo claramente influenciado por la Escuela de los Annales, lo que queda expresado en particular en la obra de Álvaro Jara (véase Guerra y sociedad en Chile, 1971 [1961]). El ámbito de renovación fue terreno fértil para la prédica del etnohistoriador John Murra quien, al menos desde 1965, mantuvo contactos con esta universidad. Como ha remarcado Jorge Hidalgo (2013), los trabajos iniciales de los etnohistoriadores chilenos debieron mucho a la influencia de este antropólogo, centrada en el reconocimiento de la complejidad de la organización social, económica y política andina, en la relación entre las sociedades y sus ecologías y en las transformaciones históricas que estos grupos experimentaron desde la conquista de América. Para Hidalgo, además, no debería dejarse de lado el momento sociopolítico del país, en particular la implementación de la Reforma Agraria, ya que, en ese contexto, el enfoque etnohistórico fue una vía que permitió explorar las dimensiones étnicas de las poblaciones campesinas.

Además, en el norte del país, espacio geográfico de las investigaciones, la Etnohistoria andina comenzó a practicarse acompañando el proceso de institucionalización del campo regional, la consecuente llegada de investigadores profesionales de otras zonas del país y la formación de cuadros académicos locales. Para este lugar, en contraste con el rico acervo arqueológico que venía rescatándose, se sufría la falta de archivos documentales. La pesquisa en este sentido fue una de las primeras tareas a la que se abocaron los investigadores (Hidalgo 2013: 244-247). El relevamiento de los artículos etnohistóricos en publicaciones de la región (v.g. Chungara, Estudios Atacameños) con posterioridad al golpe de Estado da cuenta de la evolución cuantitativa y cualitativa respecto de temáticas como demografía, economía, relaciones interétnicas, movilidad y recursos.

Frente a las perspectivas de las fuentes que analizamos en este artículo, centradas en la categoría de desintegración (a la que consideramos un obstáculo epistemológico sensu Bachelard [1973]), los desarrollos de la naciente Etnohistoria andina, al intentar enfatizar en las estrategias mediante las cuales las comunidades se habían adaptado a los cambios de época, significaron una verdadera ruptura epistemológica (sensu Bourdieu et al. 2002). La Etnohistoria, en su calidad de campo interdisciplinar, abonó los cambios experimentados por la Arqueología, al posibilitar un relacionamiento entre los registros arqueológico, documental y etnográfico; por la Historia, al redireccionar su mirada hacia los pequeños procesos y las poblaciones que habían sido negadas por las oligarquías como integrantes de la nación; y por la Antropología, al despertar el interés por el campesinado indígena en el marco de los procesos reformistas de la época (Hidalgo 2013: 243). Empezó a reconocerse así la larga historia de contacto con otras sociedades dominantes. A destacarse el aprovechamiento de los diferentes escenarios históricos. A proponerse que las estrategias étnicas de movilidad constituían un rasgo recurrente y que, desde la perspectiva indígena, estos movimientos no eran novedosos ni llevaban ineluctablemente a la desintegración. Las técnicas agrícolas, el uso de diferentes pisos ecológicos y el acceso a bienes regulados por la reciprocidad pasaron a estar en el núcleo de las investigaciones (Galdames y Ruz 2010).

De este modo, sin resignar la aplicabilidad del conocimiento así generado y abandonando viejos lastres heredados de la etapa pionera de los estudios andinos, las sociedades estudiadas empezaron a ser reposicionadas -como señaló José Luis Martínez (2004) - tal que sujetos históricos, en el intento de construir un objeto de conocimiento que las incluyeran en tanto agentes activos de la historia social chilena.

Como ha señalado Eduardo Menéndez, lo ideológico es un instrumento que constituye a la realidad y que también puede operar como 
crítica a esa realidad. Por tanto, la ponderación que el mismo ha tenido aquí ha sido con objeto de "comprender la forma en que se han constituido y usado los saberes" plasmados en nuestras fuentes (Menéndez 2002: 169). En este sentido, señalemos que nuestro delineamiento de los imaginarios reformista y revolucionario no debe conducir a una lectura pesimista. En su aplicación científica -es decir, expresándose por medio de los marcos estructural-funcionalista y marxista- es indudable que estos implicaron cierta alienación teórica. Sin embargo, en tanto los imaginarios sociales tienen un impacto concreto/performativo respecto de la realidad, estos constituyeron vehículos privilegiados de una acción que posibilitó la transformación social vivida por Chile en esos años. En lo tocante al plano científico, su conocimiento siempre es perfectible y, por tanto, todo análisis será, más tarde o más temprano, superado en parte.

\section{Agradecimientos}

Agradezco a Jorge Hidalgo, quien realizó comentarios acerca del texto original de este artículo, que fue presentado en calidad de ponencia en el IX Congreso Internacional de Etnohistoria (Arica). Estoy en deuda además con Mario Rivera por haberme permitido realizarle una entrevista que me brindó su punto de vista personal pertinente al tema tratado. Deseo dar las gracias también a los evaluadores, quienes han realizado una lectura crítica del presente trabajo.

\section{Referencias Citadas}

Ansaldi, W.

1991 La búsqueda de América latina. Entre el ansia de encontrarla y el temor de no reconocerla. Teorías e instituciones en la construcción de las ciencias sociales latinoamericanas. Cuadernos-Instituto de Investigaciones-FCS-UBA, Buenos Aires.

Bachelard, G.

1973 Epistemología. Traducido por E. Posa. Compilado por D. Lecourt. Anagrama, Barcelona.

Berdichewski, B.

2004 Alejandro Lipschutz: su visión Indigenista y Antropológica. UCSH, Santiago.

Boas, F.

1987 [1929] Anthropology and Modern Life. Dover Publications, New York.

Bonfil Batalla, G.

1972 El concepto de indio en América: una categoría de la situación colonial. Anales de Antropología 9: 105-124. UNAM, México.

Bourdieu, P; Chamboredon, J-C y Passeron, J-C.

2002 El Oficio del Sociólogo. Traducido por F. Azcurra. Siglo XXI, Buenos Aires.

Bunge, $\mathrm{M}$.

2000 La Investigación Científica: su Estrategia y su Filosofía. Siglo XXI, México.

Castoriadis, C.

1993 La Institución Imaginaria de la Sociedad. Traducido por A. Vicens Tusquets, Buenos Aires.

Chiappe, C.

2014a Política Desarrollista y Estudios Andinos en Chile (1960-1973). Tesis de licenciatura, FFyL, UBA, Buenos Aires. Director: Carlos Zanolli.

Chiappe, C.

2014b Entrevista al Dr. Mario Rivera. Universidad de Tarapacá. Arica, Chile.

Gadamer, H-G.

1988 Verdad y Método. Traducido por M. Olasagasti. Sígueme, Salamanca.
Galdames Rosas, L.

2009 Rómulo Cúneo-Vidal. Pionero de la etnohistoria andina. Chungara 41 (1): 45-49.

Galdames Rosas, L. y Ruz Zagal, R.

2010 La Junta de Adelanto de Arica y John V. Murra. Dos lecturas sobre el desarrollo andino en el norte de Chile. Chungara 42 (1): 257-270.

Godelier, M.

1978 [1968] Las Sociedades Precapitalistas. Traducido por L. Amador. Quinto Sol, México.

Gunderman, H. y González, H.

2009 Sociedades indígenas y conocimiento antropológico. Aymaras y atacameños de los siglos XIX y XX. Chungara 41 (1): 113-164.

Hidalgo, J.

2013 Historia de los pueblos andinos de Arica, Tarapacá y Atacama. En Los Estudios Andinos hoy. Práctica Intelectual y Estrategias de Investigación, compilado por C. Zanolli et al., pp. 243-276. Prohistoria, Rosario.

Jara, Á.

1971 [1961] Guerra y Sociedad en Chile. Universitaria, Santiago.

Klimovski, G.

1997 Las Desventuras del Conocimiento Científico. A-Z, Buenos Aires.

Lipschutz, A.

1937 Indoamericanismo y Raza India. Nascimento, Santiago.

Malinowski, B.

2000 [1922] Los Argonautas del Pacífico Occidental. Traducido por A. Desmonts. Altaya, Barcelona.

Marí, E.

1991 Ciencia y ética: el modelo de la ciencia martillo. Doxa 10: 319-327.

Mariátegui, J.C.

2007 [1928] Siete Ensayos de Interpretación de la Realidad Peruana. Minerva, Lima.

Martínez, J.L.

2004 Reseña bibliográfica. Historia Andina en Chile, Jorge Hidalgo L., Universitaria, Santiago, 2004, pp. 705. Chungara 36 (2): 525-534. 
Medina, J.

1882 Los Aborijenes de Chile. Gutenberg, Santiago.

Menéndez, E.

2000 La parte Negada de la Cultura. Bellatierra, Barcelona.

Núñez, L.

2013 Sobre los comienzos de los estudios andinos y sus avances actuales en el norte de Chile. En Los Estudios Andinos hoy. Práctica Intelectual y Estrategias de Investigación, compilado por C. Zanolli et al., pp. 79-122. Prohistoria, Rosario.

Palestini, S.; Ramos, C. y Canales, A.

2010 La producción de conocimiento antropológico social en Chile. Postransición: Discontinuidades del pasado y debilidades presentes. Estudios Atacameños 39: 101-120.

Rivera, M.

1973a A manera de introducción: Hacia una política de desarrollo integral del Departamento de Arica. Chungara 1-2: 11-16.

Rivera, $M$.

1973b Bases para planificar la investigación antropológicaarqueológica en el Norte Grande Chileno. Chungara 1-2: 39-48.

Stavenhagen, R.

1975 Siete tesis equivocadas sobre América latina. En F. Feder (compil.) La lucha de clases en el campo: 37-53. FCE, México.
Stern, S.

1982 Los pueblos Indígenas del Perú y el Desafío de la Conquista Española. Alianza, Madrid.

Steward, J.

1955 Theory of Culture Change: The Methodology of Multilinear Evolution. Urbana-University of Illinois Press, Champaign.

Stocking, G.

2002 Delimitando la antropología: Reflexiones históricas acerca de las fronteras de una disciplina sin fronteras. Revista de Antropología Social 11: 11-38. UCM, Madrid.

Taberna Gallegos, F. y Comisión Organizadora del I Congreso del Hombre Andino.

1996 [1973] "El rol de la sociedad andina y el tránsito al socialismo". En B. Guerrero (ed.) Homenaje a Freddy Taberna G. Escritos sobre la sociedad aymara del norte grande de Chile: 47-65. CREAR-El Jote Errante, Iquique. Valcárcel, L.

1927 Tempestad en los Andes. Minerva, Lima.

Varsavsky, O.

1969 Ciencia, Política y Cientificismo. CEAL, Buenos Aires.

Weber, M.

2002 [1922] Economía y Sociedad. Traducido por J. Medina Echavarría et al. FCE, Madrid. 
